\title{
Investigando Colaboração em Ecossistemas
}

\author{
Mariana Pinheiro, Luciana Chueri, Rodrigo Pereira dos Santos
}

\author{
Universidade Federal do Estado do Rio de Janeiro (UNIRIO) \\ Avenida Pasteur, 458 - Urca - CEP: 22290-255 - Rio de Janeiro, RJ, Brasil \\ \{mariana.pinheiro, luciana.chueri, rps\}@uniriotec.br
}

\begin{abstract}
In the current scenario, we live in an open world where businesses and organizations will only remain competitive if they learn to develop strategies for interaction and collaboration. Although collaboration has its benefits recognized within organizations, its dynamics in ecosystems brings many challenges. This paper presents an investigation on collaboration in digital, software, business, and innovation ecosystems. Challenges such as the lack of supporting platforms, the need to adapt processes, and social issues (e.g., lack of trust and leadership) were observed. This study calls researchers and practitioners to better design and develop solutions for these contexts.
\end{abstract}

Resumo. No cenário atual, se vive em um mundo aberto onde negócios e organizações só permanecerão competitivos se aprenderem a desenvolver estratégias para interação e colaboração. Apesar da colaboração ter seus benefícios reconhecidos nas organizações, a sua dinâmica em ecossistemas encontra uma série de desafios. Este artigo apresenta uma investigação sobre a colaboração em diferentes ecossistemas (digitais, de software, de negócios e de inovação). Desafios como a ausência de plataformas de apoio, necessidade de adaptação de processos e questões sociais (e.g., ausência de confiança e liderança) foram identificados. Este estudo convida ainda pesquisadores e profissionais a desenvolverem soluções para estes contextos.

\section{Introdução}

A palavra colaboração deriva do latim com e laborare significa trabalho em conjunto. Segundo Vreede e Briggs (2005), colaboração é a articulação de um esforço comum em direção a um objetivo. De modo geral, as principais vantagens da colaboração em organizações são: redução no tempo necessário para a execução de tarefas; melhoria da capacidade de resolver problemas complexos; aumento da capacidade criativa para gerar alternativas; discussão das vantagens e desvantagens de cada alternativa para selecionar aquelas mais viáveis e tomar decisões; aprendizagem; satisfação pessoal; e estímulo à inovação [Araujo e Magdaleno 2015].

Considerando o cenário atual do mundo aberto em que negócios e organizações só permanecerão competitivos se aprenderem a desenvolver a interação e colaboração entre si [Boscarioli et al. 2017], a competitividade tecnológica das empresas dependerá da capacidade de acesso ao conhecimento externo e de como conectá-lo às suas redes de colaboração [Rocha et al. 2019]. Neste sentido, tem sido explorada a utilização de plataformas para apoiar a integração de produtores e consumidores nos mais diferentes domínios de aplicação, propiciando a emergência dos ecossistemas digitais. Um 
Ecossistema Digital (ECODig) visa criar um ambiente digital para organizações ou agentes em rede que apoiam a cooperação, o compartilhamento de conhecimento e o desenvolvimento de tecnologias abertas e adaptáveis [Boley e Chang 2007].

Em um ecossistema cujo elemento central é software, uma categoria de ECODig que vem sendo amplamente estudada se refere ao ecossistema de software (ECOS), que consiste em um conjunto de atores atuando como uma unidade que interage com um mercado distribuído entre software e serviços [Jansen e Cusumano 2012]. Quando o elemento central é uma comunidade de negócio apoiada por uma base de organizações e indivíduos que interagem, tem-se os ecossistemas de negócios (ECON) [Moore 1993]. Por fim, se o objetivo da comunidade é promover a inovação por meio de Pesquisa e Desenvolvimento (P\&D) para produzir novos recursos ou valores, tem se o ecossistema de inovação (ECOI) [Autio e Thomas 2014].

Devido ao papel central no contexto de ecossistemas, é necessário compreender como a colaboração é reconhecida, quais suas características, formas de realização, benefícios e desafios. Entre os estudos existentes, como trabalho relacionado, foi identificado um mapeamento sistemático [Saleh et al. 2015] que visa relacionar ecossistemas digitais, sistemas de colaboração e redes de mídia social. Contudo, foram buscados apenas estudos que tentam relacionar os três conceitos, mas não são apresentados elementos sobre como a colaboração é percebida pelos atores no ecossistema que participam ou quais mecanismos apoiam a sua utilização.

Dessa forma, o objetivo deste estudo é investigar como a colaboração acontece em ecossistemas. Para isso, um mapeamento sistemático de literatura (MSL) a respeito da colaboração em ecossistemas foi realizado. A investigação foi realizada em quatro domínios de ecossistemas amplamente pesquisados na academia, a saber: digital, de software, de inovação e de negócio. Foram identificados 598 estudos nas principais bibliotecas digitais e oito deles atenderam aos critérios de inclusão e foram utilizados para responder às questões de pesquisa propostas. Além desta seção, o artigo está estruturado da seguinte forma: a Seção 2 apresenta o método de pesquisa; a Seção 3 traz os resultados deste estudo; a Seção 4 discorre sobre as contribuições obtidas; a Seção 5 discute as ameaças à validade; e a Seção 6 traz as considerações finais e oportunidades.

\section{Método de Pesquisa}

Um MSL fornece uma estrutura para a análise de relatórios de pesquisa e resultados que foram publicados, categorizando-os e fornecendo um resumo visual. Este estudo segue as diretrizes para a realização de estudos secundários proposta por Kitchenham e Charters (2007), Petersen et al. (2015) e Santos et al. (2019). Os estágios para a realização deste estudo foram: i) estabelecer o protocolo; ii) executar a pesquisa; iii) selecionar estudos; iv) extrair dados; e v) realizar análises.

O objetivo deste estudo foi definido com base na abordagem Goal-QuestionMetric [Basili 1992]: analisar características, métodos, modelos, técnicas, ferramentas, desafios, benefícios e atores envolvidos a fim de identificar a respeito à colaboração do ponto de vista de pesquisadores e profissionais no contexto de diferentes ecossistemas, no caso, ECODig, ECOS, ECON e ECOI. 


\subsection{Questões de Pesquisa}

Para estabelecer o escopo da pesquisa, foi definida uma questão de pesquisa (QP) principal, que expressa os objetivos deste estudo e que foi formulada seguindo os critérios específicos no PIO (Population, Intervention, Outcomes) [Pai et al. 2004], como pode ser visto na Tabela 1. Assim, a seguinte QP foi alcançada: "O que se sabe sobre colaboração em ecossistemas?". Para ajudar a responder a esta questão, as seguintes subquestões (Sub-Q) foram especificadas:

- Sub-Q1: Como a colaboração é definida em ecossistemas?

- Sub-Q2: Quais são as características apontadas para a colaboração?

- Sub-Q3: Quais são os métodos, técnicas e ferramentas propostos/utilizados?

- Sub-Q4: Como os métodos, técnicas e ferramentas são avaliados?

- Sub-Q5: Quais os fatores condicionadores de colaboração em ecossistemas?

- Sub-Q6: Quais são os benefícios e dificuldades reportados?

- Sub-Q7: Quem são os atores beneficiados?

- Sub-Q8: Quais são os desafios apontados?

Tabela 1. Critérios do PIO

\begin{tabular}{|c|l|}
\hline População & Ecossistemas Digitais; Ecossistemas de Software; Ecossistemas de Negócio; Ecossistemas de Inovação \\
\hline Intervenção & Colaboração \\
\hline Resultados & Métodos, modelos, técnicas, ferramentas, desafios, benefícios \\
\hline
\end{tabular}

Neste momento, as questões de pesquisa foram documentadas no Parsifal ${ }^{1}$, uma ferramenta online desenvolvida para apoiar pesquisadores na realização de MSL no contexto da área de Engenharia de Software e incorpora os processos de MSL sugeridos por Kitchenham e Charters (2007).

\subsection{Estratégia de Pesquisa e Fonte de Dados}

A estratégia de pesquisa utilizada envolveu buscas eletrônicas nas seguintes bibliotecas digitais: Scopus ${ }^{2}$, ACM Digital Library ${ }^{3}$, IEEE Xplore ${ }^{4}$, Science Direct ${ }^{5}$ e Engineering Village $^{6}$. Não foi usado nenhum filtro com relação a ano de publicação dos estudos.

\subsection{Definição dos Termos de Pesquisa}

Para o processo de pesquisa, foi criada uma string de busca, reunindo os termos mais relevantes que compõem o PIO (Tabela 1) e relacionando-os por operadores lógicos. Os termos foram escritos em inglês, por ser a língua largamente adotada em periódicos e conferências relacionadas ao tema da pesquisa. Os termos de resultados não foram utilizados na string de busca a fim de permitir o retorno de um maior número de estudos. A fim de obter resultados relevantes para o objetivo deste MSL, algumas iterações foram realizadas até se chegar a seguinte string utilizada:

$$
\begin{gathered}
\text { collaboration AND ("digital ecosystem *" OR "innovation ecosystem *" OR "software } \\
\text { ecosystem * OR "business ecosystem *) }
\end{gathered}
$$

\footnotetext{
${ }^{1} \mathrm{https}: / /$ parsif.al/

2 https://www.scopus.com/home.uri/

${ }^{3} \mathrm{http}: / / \mathrm{dl}$.acm.org/

${ }^{4} \mathrm{http}$ ://ieeexplore.ieee.org/Xplore/home.jsp

${ }^{5} \mathrm{http}: / /$ www.sciencedirect.com/

${ }^{6} \mathrm{https}: / / \mathrm{www}-\mathrm{engineeringvillage-com} /$
} 


\subsection{Critérios de Inclusão e de Exclusão}

Conforme Kitchenham e Charters (2007), critérios de inclusão (CI) e de exclusão (CE) foram definidos para os estudos retornados pela string de busca, descritos na Tabela 2.

\subsection{Processo de Seleção de Estudos}

O processo de seleção compreendeu seis etapas: 1) definição do escopo da pesquisa; 2) remoção de duplicatas; 3) $\mathbf{1}^{\mathbf{0}}$ filtro: análise de título, resumo e palavras-chave; 4) $\mathbf{2}^{\mathbf{0}}$ filtro: leitura da introdução e da conclusão; 5) aplicação da técnica de snowballing [Wohlin et al. 2014]; e 6) $\mathbf{3}^{\mathbf{0}}$ filtro: leitura completa. Para garantir qualidade e confiabilidade, cada estudo foi analisado por dois pesquisadores nas etapas 3 a 6 . No início, foi planejada a realização de uma avaliação dos estudos com base em critérios de qualidade, como uma etapa adicional no mapeamento. Porém, o número de estudos selecionados diretamente no assunto levou a decidir por não aplicar esta etapa.

Tabela 2. Critérios de Inclusão e de Exclusão

\begin{tabular}{|c|c|l|l|}
\hline$\#$ & Código & \multicolumn{1}{|c|}{ Descrição } \\
\hline & CI1 & O estudo menciona métodos, técnicas ou ferramentas no contexto de ECODig, ECOS, ECON e ECOI. \\
& CI2 & O estudo menciona características de colaboração no contexto de ECODig, ECOS, ECON e ECOI. \\
Critérios & CI3 & O estudo menciona dificuldades e benefícios da colaboração no contexto de ECODig, ECOS, ECON e ECOI. \\
de & CI4 & O estudo menciona desafios relacionados a colaboração no contexto de ECODig, ECOS, ECON e ECOI. \\
Inclusão & CI5 & O estudo menciona a definição de colaboração no contexto de ECODig, ECOS, ECON e ECOI. \\
& CI6 & O estudo menciona os fatores condicionadores de colaboração no contexto de ECODig, ECOS, ECON e ECOI. \\
& CI7 & O estudo menciona avaliação de métodos, técnicas ou ferramentas de colaboração para ECODig, ECOS, ECON e ECOI. \\
& CI8 & O estudo menciona os atores beneficiados no contexto de ECODig, ECOS, ECON e ECOI. \\
\hline \multirow{3}{*}{ Critérios } & CE1 & O artigo não está escrito em inglês ou em português. \\
de & CE2 & O artigo não está disponível para download abertamente ou através de IP institucional. \\
Exclusão & CE3 & Artigo duplicado que fala sobre um mesmo estudo (ou estudo relacionado) e reporta resultados anteriores. \\
& CE4 & O artigo é um livro, tutorial, editorial, resumo, pôster, painel, palestra, oficina, demonstração ou prefácio. \\
& CE5 & O artigo utilizou o termo colaboração apenas como uma referência para outros conceitos. \\
\hline
\end{tabular}

$\mathrm{Na}$ etapa 1, a busca eletrônica foi realizada com a string de busca nas bases de dados selecionadas. Os resultados recuperados foram armazenados na ferramenta Parsifal para realizar a etapa 2, quando os estudos duplicados foram removidos. Em seguida, a filtragem dos resultados ocorreu em três etapas. No primeiro filtro (etapa 3 ), os estudos retornados nas buscas foram analisados, aplicando-se os critérios de inclusão e exclusão e cuja seleção foi feita por meio da leitura dos títulos, resumos e palavraschave. No segundo filtro (etapa 4), foram lidas as seções de introdução e de conclusão dos estudos selecionados na etapa anterior, os quais foram novamente analisados com base nos critérios utilizados anteriormente.

Para tornar a pesquisa mais completa, na etapa 5, a técnica backward snowballing [Wohlin et al. 2014] foi aplicada aos estudos que foram aprovados no segundo filtro. Dessa forma, as referências bibliográficas dos estudos selecionados foram analisadas recursivamente, buscando-se os estudos por eles citados que também fossem relevantes para a pesquisa e realizando-se novamente as etapas 1 a 4 . Na etapa 6 , foi realizada a leitura completa dos estudos aprovados.

\subsection{Extração de Dados}

O processo de extração foi realizado de forma sistemática por meio do registro das informações necessárias em uma planilha contendo os seguintes campos: i) identificador do estudo (ID); ii) título, iii) autores; iv) fonte (periódico ou conferência); v) ano; vi) definição; vii) características; viii) métodos, técnicas e ferramentas; ix) avaliação; $x$ ) fatores condicionadores; xi) benefícios e dificuldades; xii) atores beneficiados e xiii) desafios apontados. Este formulário apoiou a discussão dos resultados do MSL. 


\section{Resultados}

\subsection{Visão Geral dos Estudos Selecionados}

Neste MSL, foram analisados estudos relevantes em cinco bases de dados de publicação principais. Inicialmente, foi aplicada a string de busca nos bancos de dados listados anteriormente, obtendo-se 598 artigos. Os resultados quantitativos de cada etapa são apresentados na Figura 1. Ao final, foram selecionados 8 estudos para extração de dados, que foram ordenados por ano e título, apresentados na Tabela 3. Para cada estudo, foi associado um identificador $(\mathrm{E} i)$ que foi usado para referenciar ao longo da discussão. Como tal, os estudos foram enumerados de E1 a E8.

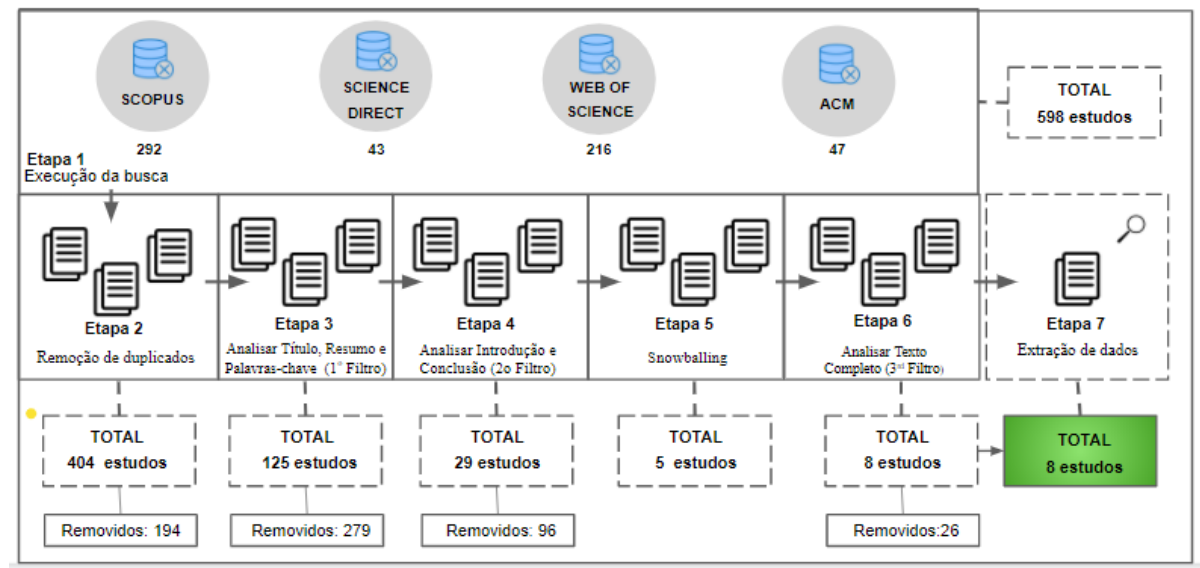

Figura 1. Resultados Quantitativos do MSL

Tabela 3. Estudos selecionados no MSL

\begin{tabular}{|c|c|l|l|c|}
\hline Ei & Ano & \multicolumn{1}{|c|}{ Título } & \multicolumn{1}{|c|}{ Autores } & \multicolumn{1}{|c|}{ Fonte } \\
\hline E1 & 2013 & $\begin{array}{l}\text { What groupware functionality do users really use? } \\
\text { A study of collaboration within digital ecosystems }\end{array}$ & Jeners, N., Lobunets, O. Prinz, W. & $\begin{array}{c}\text { IEEE International Conference on Digital } \\
\text { Ecosystems and Technologies }\end{array}$ \\
\hline E2 & 2015 & $\begin{array}{l}\text { Collaboration mechanisms for business models in } \\
\text { distributed energy ecosystems }\end{array}$ & $\begin{array}{l}\text { Hellström M., Tsvetkova A., } \\
\text { Gustafsson M., Wikström K. }\end{array}$ & Journal of Cleaner Production \\
\hline E3 & 2016 & $\begin{array}{l}\text { Collaborative Working Environments: Distance } \\
\text { factors affecting collaboration }\end{array}$ & $\begin{array}{l}\text { Pallot, M., Bergmann, U., Kuhnle, } \\
\text { H., Pawar, K.S., Riedel, J.C.K.H. }\end{array}$ & $\begin{array}{c}\text { 2010 IEEE International Technology } \\
\text { Management Conference }\end{array}$ \\
\hline E4 & 2016 & $\begin{array}{l}\text { On Baseline IT-Services to support Enterprise } \\
\text { Collaboration }\end{array}$ & Sitek, P., Sesana, M., Truong, H.-L. & $\begin{array}{c}\text { 2009 IEEE International Technology } \\
\text { Management Conference }\end{array}$ \\
\hline E5 & 2018 & $\begin{array}{l}\text { Collaboration Strategies in Innovation Ecosystems: } \\
\text { An Empirical Study of the German } \\
\text { Microelectronics and Photonics Industries }\end{array}$ & Schroth, F., Haeussermann, J.J. & $\begin{array}{c}\text { Technology Innovation Management } \\
\text { Review }\end{array}$ \\
\hline E6 & 2019 & $\begin{array}{l}\text { An observational study on the challenges faced by } \\
\text { actors in a social innovation ecosystem }\end{array}$ & $\begin{array}{l}\text { Chueri, L., Vasconcelos, A., Santos, } \\
\text { R.P. }\end{array}$ & $\begin{array}{c}\text { 11th International Conference on } \\
\text { Management of Digital EcoSystems }\end{array}$ \\
\hline E7 & 2019 & $\begin{array}{l}\text { Framework for Analysis of Multi-Party } \\
\text { Collaboration }\end{array}$ & Maksimov, Y.V., Fricker, S.A. & $\begin{array}{c}\text { 27th IEEE International Requirements } \\
\text { Engineering Conference Workshops }\end{array}$ \\
\hline E8 & 2019 & $\begin{array}{l}\text { Startups and the innovation ecosystem in Industry } \\
4.0\end{array}$ & $\begin{array}{l}\text { Rocha, C.F., Mamedio, D.F., } \\
\text { Quandt, C.O. }\end{array}$ & $\begin{array}{c}\text { Technology Analysis \& Strategic } \\
\text { Management }\end{array}$ \\
\hline
\end{tabular}

Para classificar os estudos de acordo com o tipo de contribuição, foi adotado o esquema de classificação sugerido por Abdelmaboud et al. (2015). Os tipos são descritos como: i) Ferramenta: pesquisa que apresenta uma ferramenta de software; ii) Método: pesquisa que apresenta algoritmo ou abordagem; iii) Processo: pesquisa que descreve as ações, atividades ou fluxos de trabalho associados; iv) Modelo: pesquisa que discute conceitos, faz comparações, identifica desafios ou apresenta classificações; e v) Métrica: refere-se a pesquisas que propõem medidas e métricas.

A classificação do tipo de pesquisa proposta por Wieringa et al. (2006) foi aplicada para caracterizar os estudos selecionados e o seu grau de novidade. Esta classificação é composta por seis tipos, conforme segue: i) Pesquisa de avaliação: 
investiga um problema na prática ou a implementação de uma técnica na prática; ii) Trabalho de experiência: enfatiza a experiência sobre um ou mais projetos, reflete a experiência pessoal do autor e contém uma lista de lições aprendidas; iii) Artigo de opinião: discute um tema teoricamente, ou contém uma opinião sobre o tema; iv) Artigo filosófico: esboça uma nova maneira de ver as coisas, um novo quadro conceitual etc.; v) Proposta de solução: apresenta uma solução para um problema; e vi) Pesquisa de validação: investiga as propriedades de uma proposta de solução não implementada na prática.

Como resultado dessas classificações, observou-se que as contribuições relacionadas aos modelos foram as mais expressivas (E1, E2, E3, E5, E8), uma vez que vários estudos discutiram conceitos, fizeram comparações, exploraram relações e identificaram desafios. Três estudos foram classificados na categoria de método (E4, E6, E7). Quanto ao tipo de pesquisa, seis estudos estão relacionados a pesquisas de avaliação (E1, E2, E3, E5, E6, E8) e dois deles com propostas de solução (E4, E7).

\subsection{Respostas às Questões de Pesquisa}

Após a análise dos estudos selecionados, foi possível responder às seguintes questões:

\section{- Como a colaboração é definida em ecossistemas?}

O estudo E7 citou duas definições para a colaboração em ecossistemas: i) o acordo entre especialistas para concentrar suas habilidades em um processo particular para atingir os objetivos de longo prazo de um projeto, conforme definido por um cliente [Hobbs 1996]; e ii) compromissos para integrar competências e recursos [Priego-Roche et al. 2016]. O estudo E7 apresentou a colaboração como uma composição de cinco construtos: acordo, atores, competências, objetivos, recursos e processo de colaboração.

- Quais são as características apontadas para a colaboração?

Foram identificadas as seguintes características da colaboração: i) envolve dois elementos centrais: aprendizagem interativa e a criação de novos conhecimentos (E5); ii) é multipartidária (envolve vários atores) (E7); iii) pode ser vista como um compromisso (regras registradas para trabalhar juntos, bem como para criar e trocar recursos e conhecimento); e iv) chave para o sucesso de sistemas de inovação (E5).

- Quais são os métodos, técnicas e ferramentas propostos/utilizados?

As ferramentas citadas foram: i) e-mail; ii) Confluence; e iii) GoogleDocs (E1). E6 citou a realização de reuniões e ciclos de mentoria como técnicas para incentivar colaboração entre os atores do ecossistema. Em E7, foi apresentado um framework para apoiar a elicitação de requisitos para o desenvolvimento de uma plataforma de apoio à colaboração. Este framework se baseia em métodos existentes como: Business Entities, Stakeholder Network e Inter-organisational Context.

\section{- Como os métodos, técnicas e ferramentas são avaliados?}

Diferentes formas de investigação foram citadas, a saber: i) estudos de observação (E6, E1); ii) estudos de caso (E2); iii) entrevistas semiestruturadas (E5, E7, E8); e iv) pesquisas de opinião (E3).

\section{- Quais os fatores condicionadores de colaboração em ecossistemas?}

Para E3, aumentar o número de atores colaborando leva sistematicamente a um aumento exponencial da sobrecarga de gerenciamento e integração, que por sua vez impede o 
desempenho de colaboração (paradoxo da colaboração). Para E5, alguns fatores condicionadores são: i) a experiência acadêmica e técnica; ii) termos e condições legais favoráveis entre os atores do ecossistema; iii) histórico de trabalho compartilhado bemsucedido; e iv) importância de fatores sociais, onde a confiança é descrita como o critério mais importante para a identificação de potenciais colaboradores. Em E6, foi citada também a importância do objetivo em comum e da pré-disposição dos atores em colaborar com outros no ecossistema. Para E2, para se estabelecer a colaboração entre os atores, é necessário identificar o que gera valor para os membros do ecossistema.

\section{- Quais são os benefícios e dificuldades reportados?}

Como benefícios para os atores do ecossistema, são apontados: i) integração de experiência externa com outras organizações com objetivos diferentes, descoberta conjunta de novos mercados e pesquisa de novos campos sem pressão competitiva (E5, E8); ii) maior nível de criatividade e inovação devido a uma maior diversidade de conhecimentos (E5, E8), bem como custos reduzidos e tempo de espera em otimizar soluções com base no conhecimento específico dos parceiros e nas competências essenciais; iii) atores podem se beneficiar ao obterem acesso a conhecimentos e à infraestrutura das organizações parceiras (E5, E6, E8); iv) expansão de redes profissionais (E5); e v) melhorias nas capacidades gerenciais das organizações (E8).

\section{- Quem são os atores beneficiados?}

Foram mencionados os seguintes tipos de atores beneficiados: i) organizações, instituições, investidores, agências de marketing e provedores de conhecimento (E5); ii) governo, startup e, universidade (E8); iii) proprietário de plataforma, provedores responsáveis pela interface, produtores de conteúdo e consumidores (E7); iv) gestor do ecossistema, colaborador, mentor e desenvolvedor de inovações (E6); e v) pessoas e organizações (E4).

\section{- Quais são os desafios apontados?}

Como desafios à colaboração, foram citados: i) falta de liderança clara e falta de confiança mútua (E3); ii) dificuldades nas negociações e na concordância com códigos de conduta, processos prolongados, falta de compreensão do setor de negócios, ausência de abordagens pragmáticas para o desenvolvimento de soluções, além de objetivos conflitantes como resultado de interesses diferentes (E5); iii) ausência de plataforma digital para apoio à colaboração (E6); iv) necessidade de adaptar os processos para que sejam colaborativos (E5); v) falta de critérios de tomada de decisão para colaborar (E8); vi) necessidade de ações de gestão do conhecimento (E8); vii) a maioria das ferramentas são projetadas para uso isolado (E4); viii) diversos tipos de dados não são integrados (E4); ix) dificuldade de compor diferentes ferramentas e serviços para novas necessidades de colaboração (E4); e x) as ferramentas se concentram separadamente em organizações virtuais e comunidades virtuais profissionais $(\mathrm{E} 4)$.

\section{Discussão}

Os resultados do MSL mostram que a utilização da colaboração em ecossistemas é um tema relativamente recente nas publicações. $\mathrm{O}$ estudo mais antigo foi publicado em 2013 (E1) e os outros sete estudos foram publicados no período 2013-2019, o que indica que esta pesquisa está evoluindo no tempo. Apesar de poucos estudos selecionados e da 
novidade do assunto, observou-se que nenhum apresentou uma definição nova ou adaptada ao termo colaboração e dois estudos citaram definições existentes.

A indicação de novidade também é reforçada pela análise do tipo de pesquisa, que apontou que a maioria dos estudos primários são pesquisas de avaliação (5 estudos). Não foi identificado nenhum estudo na categoria de validação de soluções. Isso reforça a ideia de que, inicialmente, as pesquisas focam em observar a colaboração em cenários reais antes de iniciar a proposição de soluções. Em relação à análise do tipo de contribuição dos estudos selecionados, identificou-se que os estudos estavam relacionados às categorias modelo ( 5 estudos) e método ( 3 estudos). Isso pode indicar uma lacuna nas categorias de 'pesquisa de validação', 'métricas' e 'processos'. Cada estudo apresentou diferentes categorias de atores beneficiados e este fato pode estar relacionado ao contexto da pesquisa, que abrangeu ecossistemas de diferentes domínios.

Observou-se que muitos estudos (E2, E5, E6, E7, E8) investigaram a colaboração em ecossistemas reais como fontes para suas pesquisas. Estas investigações foram realizadas por meio de estudos de observação, pesquisas de opinião, estudos de caso e entrevistas semiestruturadas. Por exemplo, em E5, foram realizadas entrevistas com atores de dois ecossistemas de inovação reais da Alemanha, um da indústria microeletrônica e outro da indústria fotônica, nos quais a colaboração representa um elemento chave. Estas investigações estão alinhadas com Manikas (2019), que identificou a importância do uso de ecossistemas reais existentes em pesquisa, a fim de melhorar as evidências experimentais no assunto.

Como desafios enfrentados pelos atores, foram citados desafios de cunho tecnológico (necessidade de solução tecnológica de apoio), processual (necessidade de adaptação dos processos para apoiar a colaboração) e social (importância da confiança e liderança nas equipes). Estas categorias de desafios também foram observadas em estudos sobre a colaboração em ambientes de inovação [Camarinha-Matos et al. 2015]. Com relação a mecanismos de apoio à colaboração neste contexto, houve apenas o relato de uma ferramenta, demonstrando o alinhamento com alguns desafios identificados que citavam a ausência de apoio tecnológico.

Com relação aos benefícios da colaboração, o compartilhamento de conhecimento e experiências entre os atores do ecossistema, a realização de tarefas em menos tempo e a utilização de infraestruturas de parceiros foram os mais citados. Alguns destes benefícios foram identificados em outros trabalhos sobre colaboração em organizações [Araujo e Magdaleno 2015].

\section{Ameaças à Validade}

Esta seção analisa as ameaças à validade para este estudo e considera a validade do constructo, de confiabilidade, interna e externa, segundo Petersen et al. (2015). A validade do constructo está associada ao fato dos documentos incluídos no estudo refletirem a utilização da colaboração nos ecossistemas que se pretendia investigar. A string de busca foi definida de forma inclusiva para que capturasse estudos relacionando a colaboração a ECODig, ECOS, ECON e ECOI. As bases de dados comumente utilizadas na área de Computação foram usadas neste estudo.

A validade de confiabilidade se refere à potencial repetibilidade do estudo por outros pesquisadores. O estudo aplicou uma string de busca definida, utilizou bases de dados determinísticas, utilizou uma ferramenta gratuita de suporte ao processo de MSL 
(Parsifal) e seguiu um procedimento que pode ser facilmente replicado. Desta forma, a validade de confiabilidade foi alcançada.

A validade interna se refere a problemas na análise dos dados. Essas ameaças são consideradas pequenas, uma vez que apenas estatísticas descritivas foram usadas. A validade externa diz respeito à capacidade de generalizar a partir deste estudo. A generalização não é um objetivo de um estudo de mapeamento sistemático, pois o foco está em analisar o estado de um tópico de pesquisa, bem como os trabalhos relevantes, que foram cobertos a partir de um protocolo definido.

\section{Conclusão}

A partir das pesquisas realizadas, este é o primeiro estudo com o objetivo de investigar como a colaboração é percebida na literatura no contexto de ecossistemas digitais, de software, de negócio e inovação. Para tanto, foi realizado um MSL, no qual foram selecionados 8 artigos, extraídos e analisado os dados. Os resultados revelam que as instituições acadêmicas publicaram 100\% dos artigos selecionados. No entanto, isso não significa que os estudos selecionados não tenham aplicação na prática, uma vez que a maioria deles (seis) investigou ecossistemas existentes e um trabalho realizou uma pesquisa de opinião envolvendo profissionais e pesquisadores experientes. Isto reforça a intenção do pesquisador de propor métodos ou soluções para problemas reais.

A partir deste trabalho, espera-se ajudar pesquisadores e profissionais a terem uma visão geral sobre colaboração em relação aos: i) benefícios e dificuldades percebidos pelos atores; ii) desafios enfrentados pelos atores; iii) caracterização e formas de investigação; iii) as categorias de atores beneficiados; e iv) mecanismos de apoio utilizados. As lacunas identificadas no estudo servem como caminhos para futuras pesquisas e desenvolvimentos na área de colaboração em ecossistemas.

Algumas oportunidades de trabalhos futuros foram identificadas a partir deste estudo, tais como: i) investigar os mecanismos de colaboração mencionados em um caso de ecossistema; e ii) conduzir entrevistas com atores dos ecossistemas pesquisados para investigar como os elementos identificados se relacionam com os desafios apresentados e impactam os ecossistemas, tanto positivamente quanto negativamente.

\section{Agradecimentos}

Os autores agradecem a CAPES, UNIRIO e FAPERJ (Proc. 211.583/2019).

\section{Referências}

Abdelmaboud, A. et al. (2015) "Quality of service approaches in cloud computing: A systematic mapping study", Journal of Systems and Software, 101:159-179.

Araujo, R.M., Magdaleno, A.M. (2015) "Social BPM: Processos de Negócio, Colaboração e Tecnologia Social", In: SBSI 2015, Goiás.

Autio, E., Thomas, L. (2014) "Innovation Ecosystems: Implications for Innovation Management", In: The Oxford Handbook of Innovation Management, 1(11):204-228

Basili, V. R. (2008) "Software modeling and measurement: the Goal/Question/Metric paradigm", The title of book two, 21(2):277-292. 
Boley, H., Chang, E. (2007) "Digital Ecosystems: Principles and semantics”, In: 2007 Inaugural IEEE DEST, Cairns, pp.398-403.

Boscarioli, C., Araujo, R.M., Maciel. R.S.P (2017) "I GranDSI BR Grand research challenges in information systems in Brazil 2016 2026", Special Committee on Information Systems (CESI). Brazilian Computer Society (SBC), 184 p.

Bosch, J. (2009) "From software product lines to software ecosystem", In: Proceedings of 13th International Software Product Line Conference, San Francisco, pp.1-10.

Camarinha-Matos, L.M., Bénaben F., Picard W. (2015) "Risks and Resilience of Collaborative Networks", In: 16th IFIP WG 5.5 Working Conference on Virtual Enterprises, Albi, pp.23-336.

De Vreede, G.J., Briggs, R.O. (2005) "Collaboration engineering: designing repeatable processes for high-value collaborative tasks", In: $38^{\text {th }}$ HICSS, Big Island.

Hobbs, R. W. (1996) “Leadership through collaboration”, AI Architect, 3(11)

Jansen, S., Cusumano, M. (2012) "Defining software ecosystems: A survey of software ecosystems and ecosystem", In: IWSECO, Cambridge, pp. 41-58.

Kitchenham, B., Charters, S., (2007) "Guidelines for performing systematic literature reviews in software engineering”, In: Technical Report EBSE-2007-01, Inglaterra.

Manikas, K. (2019) "Revisiting software ecosystems research: A longitudinal literature study", The Journal of Systems and Software, 117:84-103.

Moore, J. F. (1993) "Predators and Prey - A New Ecology of Competition", Harvard Business Review, 71:75-86.

Pai, M., McCulloch, M., Gorman, J., Pai, N., Enanoria, W., Kennedy, G., Tharyan, P. Colford Jr, J.M., (2004) "Systematic reviews and meta-analyses: an illustrated, stepby-step guide", The National Medical Journal of India, 17(2):86-95.

Petersen, K. Vakkalanka, S., Kuzniarz, L. (2015) "Guidelines for conducting systematic mapping studies in software engineering: An update", Information and Software Technology. 64:1-18.

Priego-Roche, L.M., Front, A., Rieu, D. (2016) “A framework for virtual organization requirements", Requirements Engineering, 21(4):439-460.

Saleh, M., Abel, M., Misséri, V. (2015) "Investigating the similarity between collaboration systems and digital ecosystems", In: 19th IEEE CSCWD, Calabria, pp.30-35.

Santos, M, Fernandes, J., Villela, M., Santos. R.P (2019) "Human computer interaction factors in software ecosystems: A systematic mapping study", In: SBSI'19, New York.

Wohlin, C. (2014) "Guidelines for snowballing in systematic literature studies and a replication in software engineering", In: 18th International Conference on Evaluation and Assessment in Software Engineering, New York, pp.1-10

Wieringa, R., Maiden, N, Mead, N., Rolland, C. (2006) "Requirements engineering paper classification and evaluation criteria: a proposal and a discussion", In: Requirements engineering 11, London, pp.102-107. 